\title{
Experience of percutaneous access under ultrasound guidance in renal transplant patients with allograft lithiasis
}

\author{
Silvano Palazzo, Ottavio Colamonico, Saverio Forte, Matteo Matera, Giuseppe Lucarelli, Pasquale \\ Ditonno, Michele Battaglia, Pasquale Martino \\ Department of Emergency and Organ Transplantation, University of Bari, Italy.
}

\begin{abstract}
Summary Objective: Urolithiasis of the transplanted kidney has an incidence of 0.2 to $1.7 \%$, it increases the risk of infection in immunosuppressed patients and it can lead to ureteral obstruction that is often associated with deterioration of renal function. Urolithiasis of the transplanted kidney has different characteristics compared to the native kidney, due to the absence of innervation, which does not lead to colic pain. Percutaneous approach is an optimal choice in transplant patients.

Material and methods: Here we report our experience in two cadaveric transplant patients with urolithiasis. The first case was a patient of 68 years with a $20 \mathrm{~mm}$ stone located in the transplanted kidney pelvis and another smaller in a lower calyx. The second case was a patient of 65 years with a 15 $\mathrm{mm}$ stone in the distal part of the transplanted ureter. In both cases the patients were asymptomatic, but they had a reduction in urine output associated with worsening of the transplanted kidney function. The diagnosis was performed in both cases with ultrasound study, showing a severe hydronephrosis and it was confirmed by computed tomography scan. In both cases, we performed a Percutaneous Nephrolithotomy (PCNL). Access was made after targeting the stone, through a lower pole puncture under ultrasound guidance. The first case was treated with pneumatic and laser energy, breaking stones through a nephroscope. In the second case we performed a laser lithotripsy of the ureteral stone, using a flexible videoureteroscope. At the end of both procedures a Double-J stent and a $14 \mathrm{Fr}$ Malecot nephrostomy were positioned, that were removed at 6 weeks and 10 days, respectively.

Results: Both patients achieved a resolution of the worsening of renal function, recovering the spontaneous diuresis. The surgical procedure using ultrasound guidance was safe and allowed quick access to the renal pelvis. Both patients experienced no bleeding or infection during hospitalization. Conclusions: Percutaneous Nephrolithotomy (PCNL) is an established safe and effective surgical treatment option for larger renal calculi in renal allografts. The ultrasound guided access to the transplanted kidney in percutaneous treatment of urolithiasis is useful and fast, minimizing patient exposure to ionizing radiation.
\end{abstract}

KEY WORDS: Renal transplant; Urinary calculi; Percutaneous nephrolithotomy.

Submitted 15 November 2016; Accepted 15 December 2016

\section{INTRODUCTION}

Urolithiasis of the transplanted kidney is a rare complication that occurs in $0.4-1 \%$ of the population of trans- planted patients (1). Although it represents a rather uncommon complication, it can lead to significant morbidity and to a devastating loss of graft function if obstruction occurs (2). Described for the first time in 1970 by Rattiazzi and associates (3), the urolithiasis of the transplanted kidney is considered a transplant complication deserving of a minimally invasive surgical approach, such as percutaneous nephrolithotomy (PCNL) (4). In fact PCNL is the technique of choice for removal of stones larger than $1.5 \mathrm{~cm}$ in diameter and it has the advantage of potentially removing all stone fragments at one procedure (5). Among the 1291 transplants performed in our institution, 10 (0.7\%) showed nephrolithiasis in the renal graft, but only 4 have necessitated surgery. In this report, we describe two patients with kidney transplantation and urolithiasis treated with PCNL under ultrasound guidance.

\section{Case report}

The first case was a 68 year old male patient that had received a cadaveric kidney transplant, in the right iliac fossa, 16 years previously for end-stage renal disease of unknown etiology. In 2012 he has undergone pyelolithotomy for transplanted kidney stones before ureteral reimplantation according to Lich-Gregoir technique. The patient was in pharmacological therapy for hypertension and type 2 diabetes. He came to our attention for anuria and acute renal failure secondary to a severe hydroureteronephrosis of the allograft (documented by an ultrasound scan) in absence of colic pain. Therefore the patient was drained with a percutaneous nephrostomy, that was removed after a few days when it was placed an anterograde Double-J ureteral stent. Then a computed tomography scan revealed a $20 \mathrm{~mm}$ stone located in the transplanted kidney pelvis and another smaller in a lower calyx (Figures 1A, 1B). Subsequently, after refusing endoscopic surgery to remove obstruction, he underwent a single session of ESWL that remained unsuccessful. After this failure, the patient decided to undergo PCNL. Therefore, PCNL treatment was carried out in the supine position and general anesthesia. At first, a lower pole puncture was performed, under ultrasound guidance, in order to introduce a stiff 0.038 " guidewire into the pelvicalyceal system. A skin incision along the guidewire was made and dilation was performed using a balloon dilator. After tract dilation, 


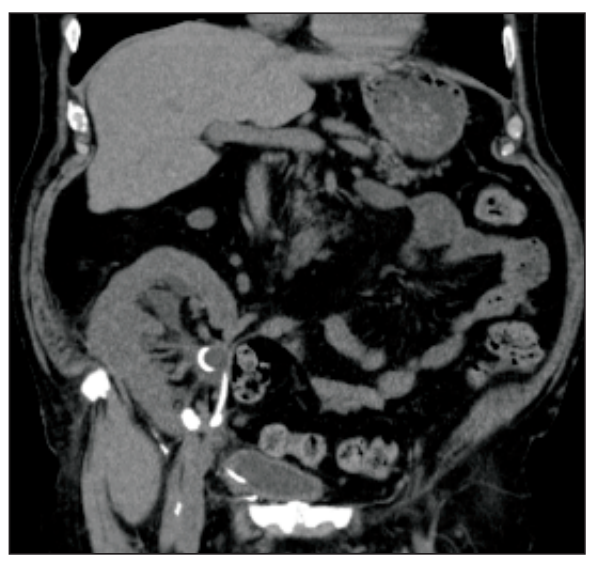

Figure 1A.

Coronal

computed

tomography

image showing

stone in a

lower calyx and

the double-J

stent in the

right iliac fossa

renal allograft.

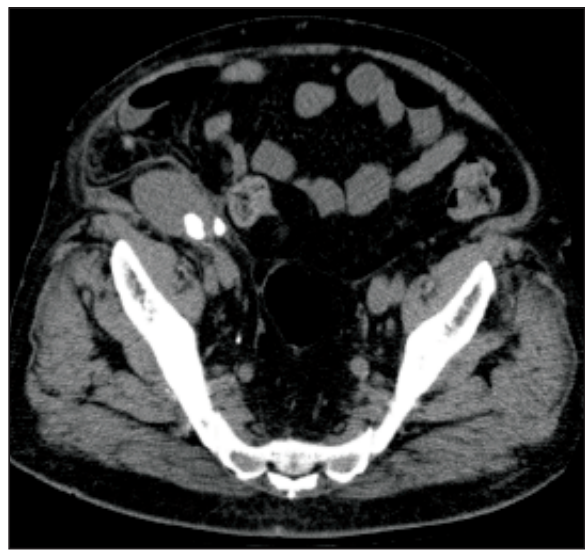

Figure 1B.

Axial

(transverse)

computed

tomography

image showing

2 stones in the

right iliac fossa

renal allograft

(the first

located in the

kidney pelvis

and another

smaller in a

lower calyx). the percutaneous sheath was advanced into the pelvicalyceal system. Dilation and advancement of the operating sheath was accomplished under X-ray control. A rigid nephroscope was introduced through the operating sheath and an Holmium laser lithotripsy using $365 \mu \mathrm{m}$ laser fibers was performed. During the procedure we also used a pneumatic lithotriptor (Stone Breaker). Both stones were destroyed and the fragments were removed with graspers. Finally a temporary percutaneous 14 Fr Malecot nephrostomy was positioned in the renal pelvis, also leaving the previously positioned ureteral stent.

The second case was represented by a 65 year old male patient with a medical history of hypertension and chronic kidney disease secondary to ADPKD (Autosomal Dominant Polycystic Kidney Disease).

In 2009 he was subjected to cadaveric kidney transplantation in the right iliac fossa but after few months occurred a ureteral stricture that required ureteral reimplantation according to Lich-Gregoir technique. This patient was admitted to our department for a progressive loss of the allograft function associated with severe hydroureteronephrosis (diagnosed through an ultrasound scan). Before surgery was performed an abdomen computed tomography scan which revealed the presence of a $15 \mathrm{~mm}$ stone in the distal part of the transplanted ureter (Figures 2A, 2B). Therefore, the patient was prepared for PCNL procedure. At first, the patient was positioned supine and we made a lower pole puncture, under ultrasound guidance, in order to introduce a percutaneous nephrostomy into an anterior calyx of the
Figure $2 A$.

Axial (transverse) computed tomography image showing stone in the distal part of the transplanted ureter.
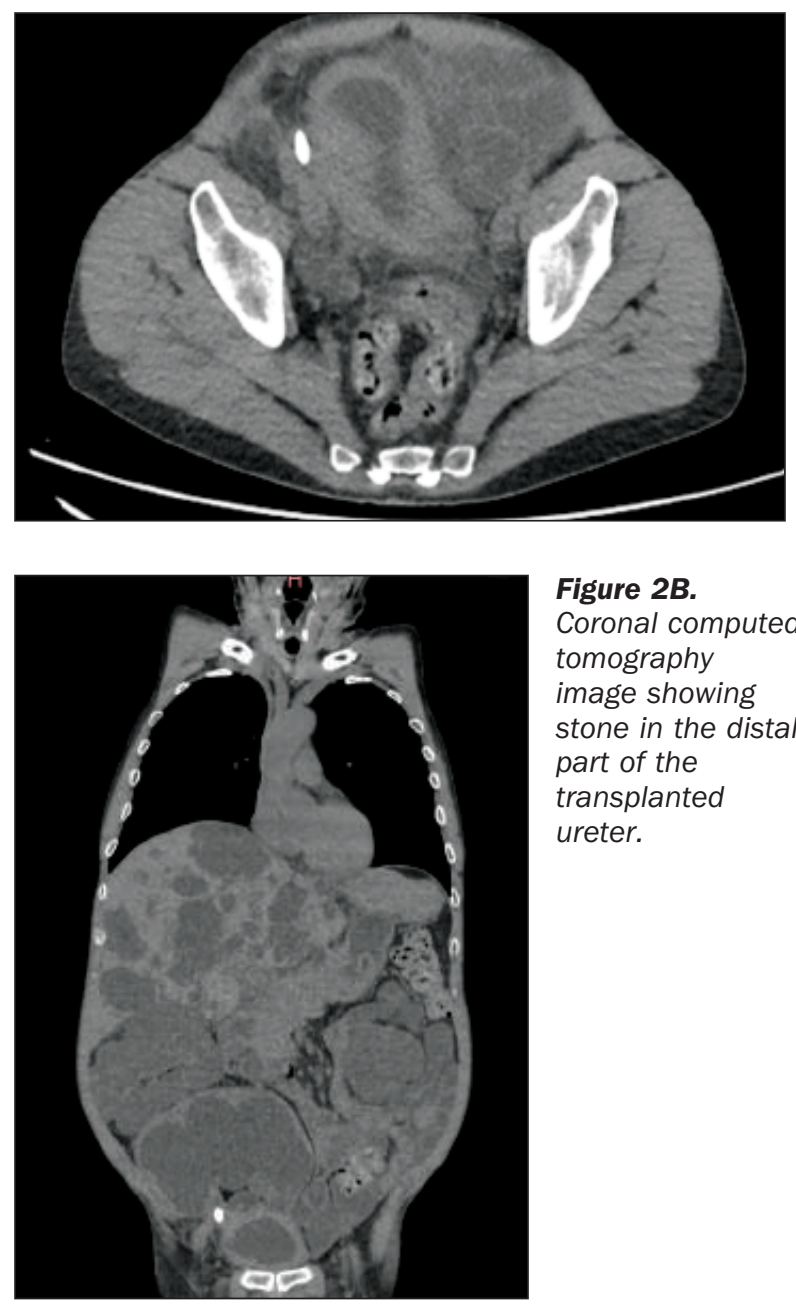

Figure 2B.

Coronal computed tomography image showing stone in the distal part of the transplanted ureter.

transplanted kidney. Then we made an anterograde ureteropyelography, under X-ray control, which confirmed the presence of a partial obstruction located in the distal part of the allograft ureter. After putting the guidewire through the nephrostomy, the nephrostomy was removed and we proceeded to dilate the operating channel in order to introduce the percutaneous sheath (always under X-Ray control). So that we performed a Holmium laser lithotripsy of the ureteral stone, through a flexible video- ureteroscopy. Stone fragments were removed with graspers. Finally we introduced an anterograde Double-J stent in order to prevent ureteral stricture, but also a temporary percutaneous 14 Fr Malecot nephrostomy. After surgery both patients immediately had an improvement of renal function with a postoperative course uneventful, experimenting minimal pain. In particular they had no bleeding or infection during hospitalization.

In both cases percutaneous nephrostomy was removed only after 10 days under X-ray control, after contrast injection by the nephrostomy which confirmed a stone free state. Ureteral stents were removed cystoscopically after 6 weeks. 


\section{Discussion}

Urinary lithiasis, following renal transplantation, represents a rather uncommon complication $(0.4-1 \%)(1,6)$. More than one-half of the patients with stones in a kidney transplantation don't show any symptom of pain. The plausible explanation for this observation is denervation of the transplanted graft (7). The most common symptoms are hematuria, oliguria/anuria, fever, elevation of serum creatinine level, and positive findings in bacterial culture of urine (8). Therefore urinary stones can be regarded a potential threat due to the risk of obstruction, sepsis, and potential loss of allograft function (9).

Allograft stones may be already placed in situ, the socalled donor-gifted allograft lithiasis, or may be the result of new stone formation after transplantation. The latter is believed to be a multifactorial phenomenon, related to both metabolic and urodynamic parameters (10).

Predisposing risk factors for stone formation are present in most renal allograft recipients. Metabolic factors predisposing to stone formation are hyperparathyroidism, hypercalciuria, recurrent urinary tract infection, hypocitraturia and gout. Less common risk factors include outflow obstruction, foreign bodies such as stents, nephrostomy tubes, suture materials and donor lithiasis $(11,12)$. Harper et al. investigated risk factors for stone formation in five patients and compared them to 41 transplant patients with no stones. They reported that although patients with calculi in transplants passed significantly more concentrated and alkaline urine, there were other factors contributing to stone formation (13). The treatment methods of urolithiasis in renal transplant recipients are similar and equally safe as those used in the general population. The options for management of transplant stones are conservative, ESWL, percutaneous nephrolithomy (PCNL), ureteroscopy (URS) or open surgery. In particular Percutaneous removal of calculi from transplanted kidneys, since it was first described in 1985 by Hulbert et al. (13), is the technique of choice for removal of stones larger than $1.5 \mathrm{~cm}$ in diameter and it has the advantage of potentially removing all stone fragments at one procedure (5). Furthermore, because of the relative superficial position of transplanted kidneys, percutaneous management is favored. Indeed PCNL has the best success rate, but it is an invasive procedure on a single functioning kidney (14). Krambeck, who described the largest series of PCNL in transplant kidneys, reported perioperative complications in about $1 / 4$ of patients (15). These complications, however, were to be ascribed more to the state of immunosuppression that surgery. After these considerations, we must consider the role of ultrasonography in the management of kidney transplant complications, such as urolithiasis. In fact, ultrasonography appears to be the most useful diagnostic tool to detect stones and determine their location and size. Furthermore it is an easily applied bedside examination that can guide our intervention, in order to treat this complication $(16,17)$. When we perform a PCNL, the allograft is located in the iliac fossa and the access is typically by way of an anterior calyx with the patient in a supine position. Intra-operative ultrasound can be used to assess anatomy before access is attempted, because the proximity of bowel loops and iliac blood vessels intro- duce the possibility of injury to these structures. In addition immunosuppressive therapy can increase the risk of complications particularly impaired wound healing, fistula formation and perinephric urinoma (5).

The aim of our report is to stress the importance of ultrasonography in percutaneous treatment of the transplanted kidney lithiasis. Ultrasound has a limited application in percutaneous treatment of native kidney stones because we have some anatomical landmarks (the inferior border of the $12^{\text {th }}$ rib, iliac crest, posterior axillary line) that allow to minimize the risk of damage to the abdominal organs and vascular structures. Instead, although the transplanted kidney has a more superficial location, there is an increased risk of bowel damage and injury to the iliac vessels. Since the transplanted kidney has an extra anatomical location, there are no bone landmarks capable of guiding the operator in the proper approach to the urinary tract.

The extra anatomical position of the neo-ureteral meatus, in association with the difficult accessibility to the high urinary tract because of perimeatal and periureteral fibrosis, make complex if not impossible to execute an ascending pyelography, preventing an X-ray guided approach to the transplanted kidney (18). For this reason we believe that ultrasound can speed up the percutaneous access to the kidney transplant for the treatment of urolithiasis, reducing the risk of bowel and vascular damage. In addition ultrasound, reducing surgery time, may decrease the patient's exposure to ionizing radiation.

\section{Conclusion}

The formation of urinary stone following renal transplantation is a rare complication. Percutaneous Nephrolithotomy (PCNL) is an established safe and effective surgical treatment option for larger renal calculi in renal allografts. In our opinion, the ultrasound guided access to the transplanted kidney in percutaneous treatment of urolithiasis is useful and fast, minimizing patient exposure to ionizing radiation.

\section{REFERENCES}

1. Rhee BK, Bretan PN Jr, Stoller ML. Urolithiasis in renal and combined pancreas/renal transplant recipients. J Urol. 1999; 161:1458-62.

2. Stravodimos KG, Adamis S, Tyritzis $S$, et al. Renal transplant lithiasis: analysis of our series and review of the literature. J Endourol. 2012; 26:38-44.

3. Rattiazzi LC, Simmons RL, Markland C, et al. Calculi complicating renal transplantation into ileal conduits. Urology. 1975; 5:29-31.

4. Challacombe B, Dasgupta P, Tiptaft R, et al. Multimodal management of urolithiasis in renal transplantation. BJU Int. 2005; 96:385-389.

5. Wong KA, Olsburgh J. Management of stones in renal transplant. Curr Opin Urol. 2013; 23:175-9.

6. Mamarelis G, Vernadakis S, Moris D, et al. Lithiasis of the renal allograft, a rare urological complication following renal transplantation: a single-center experience of 2,045 renal transplantations. Transplant Proc. 2014; 46:3203-5. 
7. Ferreira Cassini M, Cologna AJ, Ferreira Andrade M, et al. Lithiasis in 1,313 kidney transplants: incidence, diagnosis, and management. Transplant Proc. 2012; 44:2373-5.

8. Li SD, Wang QT, Chen WG. Treatment of urinary lithiasis following kidney transplantation with extracorporeal shock-wave lithotripsy. Chin Med J (Engl) 2011; 124:1431e4.

9. Verrier C, Bessede T, Hajj P, et al. Decrease in and management of urolithiasis after kidney transplantation. J Urol. 2012; 187:1651e5.

10. Crook TJ, Keoghane SR. Renal transplant lithiasis: Rare but time-consuming. BJU Int. 2005; 95:931-933.

11. Klingler HC, Kramer G, Lodde M, Marberger M. Urolithiasis in allograft kidneys. Urology. 2002; 59: 344-8.

12. Rubio BJ, Chechille TG, Parada MR, et al. Lithiasis in renal transplantation. Actas Urol Esp. 1995; 19:561-565.
13. Hulbert JC, Reddy P, Young AT, et al. The percutaneous removal of calculi from transplanted kidneys. J Urol. 1985; 134:324-6.

14. Markic D, Krpina K, Ahel J, et al. Treatment of Kidney Stone in a Kidney-Transplanted Patient with Mini-Percutaneous Laser Lithotripsy: A Case Report. Case Rep Nephrol Dial. 2016; 6:26-31.

15. Krambeck AE, Leroy AJ, Patterson DE, Gettman MT. Percutaneous nephrolithotomy success in the transplant kidney. J Urol. 2008; 180:2545-9.

16. Hyang K, Jhoong S. Cheigh, Hee Won Ham. Urinary Stones following Renal Transplantation. Korean J Intern Med. 2001; 16:118122.

17. Kolofousi C, Stefanidis K, Cokkinos DD, et al. Ultrasonographic Features of Kidney Transplants and Their Complications: An Imaging Review. ISRN Radiol 2013; 2013:480862.

18. Lu H, Shekarriz B, Stoller ML. Donor-gifted allograft urolithiasis: early percutaneous management. Urology. 2002; 59:25.

\section{Correspondence}

Silvano Palazzo, MD

Ottavio Colamonico, $\mathrm{MD}$

Saverio Forte, MD

Matteo Matera, MD

Giuseppe Lucarelli, MD

Pasquale Ditonno, MD

Michele Battaglia, MD

Pasquale Martino, MD

Department of Emergency and Organ Transplantation,

University of Bari, Bari, Italy 